\title{
3-\$32-3 Symposium32
}

\section{Development of anti-cancer agent targeting on (pro)renin receptor}

\section{Akira Nishiyama, Asadur Rahman}

\author{
Dep. Pharmacology, Kagawa Med. School
}

We previously reported that silencing of the $P R R$ gene, which encodes the (pro)renin receptor $((\mathrm{P}) \mathrm{RR})$ significantly reduced Wnt/ $\beta$-catenin-dependent development of pancreatic ductal adenocarcinoma(PDAC), colorectal cancer and glioblastoma. Here, we examined the effects of a panel of blocking monoclonal antibodies (mAbs) directed against the (P)RR extracellular domain on proliferation of the human PDAC cell lines PK-1 and PANC-1 in vitro and in vivo. We observed that four rat anti-(P)RR mAbs induced accumulation of cells in the G0/G1 phase of the cell cycle and significantly reduced proliferation in vitro concomitant with a significant reduction in the expression of active $\beta$ catenin and cyclin D1. Systemic administration of the anti-(P)RR mAbs to nude mice bearing subcutaneous PK-1 xenografts significantly decreased tumor expression of active $\beta$-catenin and the proliferation marker Ki-67 and reduced tumor growth. In contreffects ast, treatment with the handle region peptide of (pro)renin did not inhibit tumor growth in vitro or in vivo, indicating that the effects of the anti-(P)RR mAbs was independent of the reninangiotensin system. These data indicate that mAbs against human $(\mathrm{P}) \mathrm{RR}$ can suppress PDAC cells proliferation by hindering activation of the Wnt/ $\beta$-catenin signaling pathway. Thus, mAb-mediated (P)RR blockade could be an attractive therapeutic strategy for PDAC and other cancers. 